\title{
Wireless Oscilloscope using Android App for Mobile
}

\author{
Sreenivasan. $\mathbf{R}^{1}$, Jayarani. $\mathbf{E}^{2}$ \\ Department of ECE, Angel College of Engineering and Technology ${ }^{1}$ \\ Department of CSE, Angel College of Engineering and Technology ${ }^{2}$
}

\begin{abstract}
The Wireless Oscilloscope android application helps one to observe the waveform of the closed circuit. This application is supported by all Smartphone's and tablet computers (Receiver's) that work on Android operating system. Hence it is easy portable and easy installation. At the end of the closed circuit, Wireless Fidelity (Wi-Fi) Module is used to transmit the waveform signal over radio frequency and then is received by receiver using Wi-Fi. At last Graphical Waveform is reproduced in receivers
\end{abstract}

Keywords: Cathode-Ray Oscilloscope, Wireless Fidelity, Voltage Divisions (Voltage Div),Time Divisions(Time Div), Graphical waveform of output waves, Frequency, IEEE, Mobility, Android Application, Linux-Based operating system and Graphical waveform.

\section{INTRODUCTION}

In the modern world, Cathode-Ray Oscilloscope (CRO) plays a major role in electronics measurement filed. CRO is mainly used to measure the voltage across the circuit with variation in time. CRO prices basically from $\$ 475$ (22,500 Indian-Rupees). CRO is mostly used for experimental purposes in educational institutions and official labs. The major disadvantages of CRO are to spend huge amount of money to buy it and non-easy mobility. To overcome this, CRO functions can be brought as an Android application (using java).Android is a Linux-based operating system designed basically for touch screen mobile devices such as Smartphone's and tablet computers. Android application is an application which works on Android platform. For example, waveform across the circuit needs to be obtained graphically. Then circuit is closed with a Wireless Fidelity (Wi-Fi). Wi-Fi transmitter is used to transmit the waveform as in form of data through IEEE 802.11.1 to 802.11.4 standards. The resultant waveform is obtained in smart phones by receiving the transmitted data via Wi-Fi. Then data is reproduced as graphical waveform. Using this waveform, voltage is measured across the circuit. Advantage of this Android application is mainly Ecological Sustainable Application, high Mobility, three times lesser than the cost of CRO and Wireless (works up-to 10-100m).

\section{CATHODE RAY OSCILLOSCOPE}

The Cathode Ray Oscilloscope (CRO) is a type of electronic test instrument that allows observation of constantly varying signal voltages, usually as a twodimensional graph of one or more electrical potential differences using the vertical or $y$-axis, plotted as a function of time (horizontal or $x$-axis). Many signals can be converted to voltages and displayed this way. Signals are often periodic and repeat constantly so that multiple samples of a signal which is actually varying with time are displayed as a steady picture. This allows the measurement of peak-to-peak voltage of a waveform, the frequency of periodic signals, the time between pulses, the time taken for a signal to rise to full amplitude (rise time), and relative timing of several related signals. Before the advent of digital electronics oscilloscopes used cathode ray tubes as their display element (hence were commonly referred to as CROs)

\section{GENERAL EXTERNAL EXPERIENCE}

3.1. Display

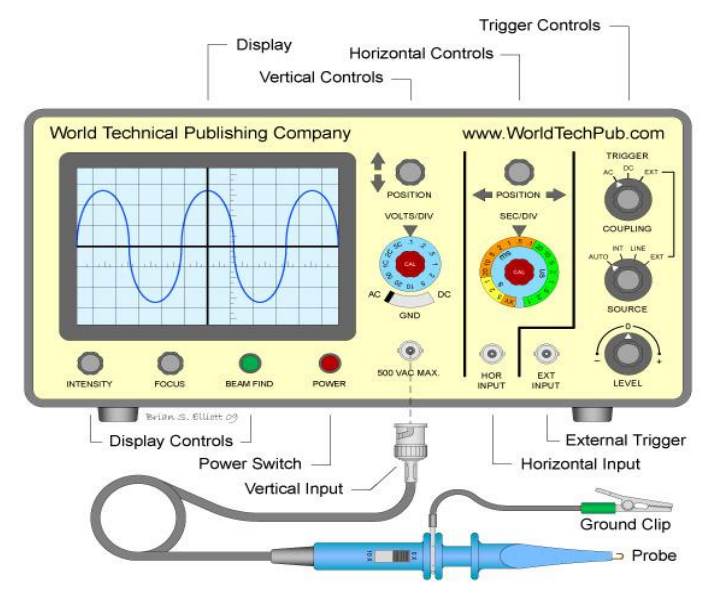

Fig 1: Basic Oscilloscope

The basic oscilloscope, as shown in the illustration, is typically divided into four sections: the display, vertical controls, horizontal controls and trigger controls

The vertical section controls the amplitude of the displayed signal. This section carries a Volts-perDivision (Volts/Div) selector knob, an AC/DC/Ground selector switch and the vertical (primary) input for the instrument. Additionally, this section is typically equipped with the vertical beam position knob.

The horizontal section controls the time base or "sweep" of the instrument. The primary control is the Seconds-per-Division (Sec/Div) selector switch. Also included is a horizontal input for plotting dual $\mathrm{X}$-Y axis signals. The horizontal beam position knob is generally located in this section. 
The trigger section controls the start event of the sweep. The trigger can be set to automatically restart after each sweep or it can be configured to respond to an internal or external event. The principal controls of this section will be the source and coupling selector switches. An external trigger input (EXT Input) and level adjustment will also be included.

\subsection{Size and portability}

Most modern oscilloscopes are lightweight, portable instruments that are compact enough to be easily carried by a single person. In addition to the portable units, the market offers a number of miniature batterypowered instruments for field service applications. Laboratory grade oscilloscopes, especially older units which use vacuum, are generally bench-top devices or may be mounted into dedicated carts. Special-purpose oscilloscopes may be mounted or permanently mounted into custom instrument housing.

\subsection{Inputs}

The signal to be measured is fed to one of the input connectors, which is usually a coaxial connector such as a BNC or type. Binding or banana may be used for lower frequencies. If the signal source has its own coaxial connector, then a simple coaxial cables used; otherwise, a specialized cable called a "scope probe", supplied with the oscilloscope, is used.

\subsubsection{Vertical position control}

The vertical position control moves the whole displayed trace up and down. It is used to set the noinput trace exactly on the center line of the graticule, but also permits offsetting vertically by a limited amount. With direct coupling, adjustment of this control can compensate for a limited DC component of an input.

\subsubsection{Horizontal position control}

The horizontal position control moves the display sidewise. It usually sets the left end of the trace at the left edge of the graticule, but it can displace the whole trace when desired. This control also moves the $\mathrm{X}-\mathrm{Y}$ mode traces sidewise in some instruments, and can compensate for a limited DC component as for vertical position.

\subsection{Applications of CRO}

Oscilloscopes are used in the sciences, medicine, engineering, and telecommunications industry. General-purpose instruments are used for maintenance of electronic equipment and laboratory work. Specialpurpose oscilloscopes may be used for such purposes as analyzing an automotive ignition system or to display the waveform of the heartbeat as an electrocardiogram. Some computer sound software allows the sound being listened to be displayed on the screen as by an oscilloscope.

\section{DISADVANTAGES OF CATHODE RAY OSCILLOSCOPE}

$\checkmark \quad$ CRO uses Cathode ray tube (CRT) as their output display. The cathode ray tube (CRT) is a vacuum tube containing one or more electron guns(a source of electrons or electron emitter) and a fluorescent screen used to view images.

travel distances.

\section{- $\quad$ Large size and weight}

- High power consumption. On average, a CRT monitor consumes $2-10 x$ the power that an identically sized LCD monitor would consume, depending on the type of backlight used in the LCD screen, and its brightness setting

- A lot of heat can be emitted during operation, due to relatively high power consumption.

- Hazardous to repair/service without proper training and precautions taken.

- The glass envelope contains toxic lead and barium as X-ray radiation shielding. The phosphors can also contain toxic elements such as cadmium. Many countries treat CRTs as toxic waste and prohibit their disposal in landfills or by incineration.

$\checkmark \quad$ Reproducing the waveform observed on the graph is not much easier (i.e.) exact waveform produced can't be reproduced as image.

$\checkmark \quad$ The CRO is not easy for portable purpose.

\section{EXISTING SYSTEM TO OVERCOME THESE DISADVANTAGES}

\subsection{Existing System}

To overcome these disadvantages a Bluetooth android oscilloscope application is developed in android 2.1 using Bluetooth Transmission in 2010.In which Bluetooth transmitter is used to transmit the waveform in radio frequency and android mobile act as receiver in which android application is used to receive transmitted signal and reproduce as a waveform in moving pictures. The transmitter consists of dsPIC33FJ16GS504 (for the analog-to-digital conversion) and LMX9838 (Bluetooth SPP module).

\subsection{Existing System's Disadvantages}

- Signal is transferred from transmitter to receiver by Bluetooth Transmission. Hence only it works with in $10 \mathrm{~m}$ only.

- Data Transmission is slow (only $723 \mathrm{kbps}$ )

- Graphical waveform only can be seen and cannot be saved as a video clip or screen shot.

This application is not promoted or not yet brought to the market.

\subsection{Transmitter}

\section{PROPOSED SYSTEM}

Transmitter consists of a Wi-Fi module and Atmega8 Microcontroller, which is described below.

6.1.1 Wi-Fi

$\mathrm{Wi}-\mathrm{Fi}$, also spelled Wifi or WiFi, is a popular technology that allows an electronic device to exchange data or connect to the internet wirelessly using radio. The Alliance defines Wi-Fi as any "wireless local area network(WLAN) products that are based on the Institute of Electrical and Electronics Engineers' (IEEE)standards". Many devices can use Wi-Fi, e.g. 
personal computers, video-game consoles, An example is a portable ECG device monitoring a smartphones, some digital cameras, tablet and digital patient at home. This Wi-Fi-enabled device can audio players. These can connect to a network resource such as the Internet via a wireless network access point. Such an access point (or hotspot) has a range of about 20 meters (65feet) indoors and a greater range outdoors. Hotspot coverage can comprise an area as small as a single room with walls that block radio waves, or as large as many square miles achieved by using multiple overlapping access points.

\subsubsection{Wi-Fi Range}

Wi-Fi networks have limited range. A typical wireless access point using802.11bor802.11gwith a stock antenna might have a range of $35 \mathrm{~m}$ (120ft.) indoors and $100 \mathrm{~m}$ (300ft.) outdoors. IEEE 802.11n, however, can more than double the range. Range also varies with frequency band. Wi-Fi in the $2.4 \mathrm{GHz}$ frequency block has slightly better range than Wi-Fi in the $5 \mathrm{GHz}$ frequency block which is used by $802.11 \mathrm{a}$ and optionally by $802.11 \mathrm{n}$. On wireless routers with detachable antennas, it is possible to improve range by fitting upgraded antennas which have higher gain in particular directions. Outdoor ranges can be improved to many kilometers through the use of high gain antennas at the router and remote device(s). In general, the maximum amount of power that a Wi-Fi device can transmit is limited by local regulations, such as FCC Part 15in the US.

\subsubsection{Advantages of $\mathrm{Wi}-\mathrm{Fi}$}

- Wi-Fi allows cheaper deployment of Local Area Networks (LANs). Also spaces where cables cannot be run, such as outdoor areas and historical buildings, can host wireless LANs.

- $\quad$ The price of chipsets for Wi-Fi continues to drop, making it an economical networking option included in even more devices.

The mobile phones have any standard Wi-Fi device will work anywhere in the world.

- Access encryption (WPA2) is considered secure, provided a strong passphrases used. New protocols for quality (WMM) make Wi-Fi more suitable for latency-sensitive applications (such as voice and video). Power saving mechanisms (WMM Power Save) extends battery life.

6.1.4 Wi-Fi module

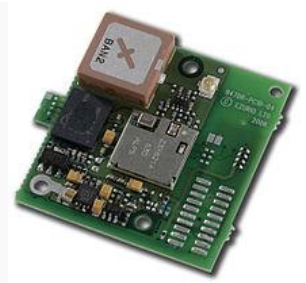

Fig2: Embedded serial-to-Wi-Fi module

Increasingly in the last few years (particularly as of 2007), embedded Wi-Fi modules have become available that incorporate a real-time operating system and provide a simple means of wirelessly enabling any device which has and communicates via a serial port. This allows the design of simple monitoring devices. communicate via the Internet.

These Wi-Fi modules are designed by OEMs so that implementers need only minimal Wi-Fi knowledge to provide Wi-Fi connectivity for their products.

6.1.5 Interfacing Wi-Fi module with Atmega8 Microcontroller

Wi-Fi module and microcontroller can be interfaces using UART. A Universal Asynchronous Receiver/Transmitter (UART) is a piece of computer hardware that translates data between parallel and serial forms. UARTs are commonly used in conjunction with communication standards such asEIA,RS-232,RS422orRS-485.A UART is usually an individual (or part of an)integrated circuit used for serial communications over a computer or peripheral device serial port. UARTs are now commonly included in microcontrollers. In fig.3; The FT232R is a USB to serial UART interface used to interface Wi-Fi module and Atmega8 microcontroller, which act as a Transmitter.

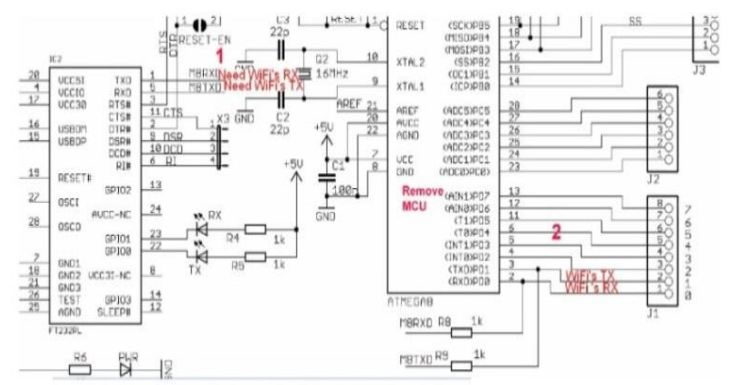

Fig3: Interfacing Wi-Fi module with microcontroller

\subsection{Receiver}

Transmitted signal is received by a smartphone's and tablet computers through Wi-Fi which works with android operating system.

6.2.1 Android

Android is a Linux-based system designed primarily for touch screen mobile devices such as smartphones and tablet. Android is open source and Google releases the code under the License. This open-source code and permissive licensing allows the software to be freely modified and distributed by device manufacturers, wireless carriers and enthusiast developers. Additionally, Android has a large community of developers writing applications ("apps") that extend the functionality of devices, written primarily in a customized version of the Java programming language. In October 2012, there were approximately 1,000,000 apps available for Android, and the estimated number of applications downloaded from Google, Android's primary app store, was 50 billion as of Sep 2013.A developer survey conducted in April-May 2013 found that Android is the most popular platform for developers, used by $71 \%$ of the mobile developer population.

These factors have contributed towards making Android the world's most widely used Smartphone 
platform, overtaking Symbian in the fourth quarter of 2010 , and the software of choice for technology companies who require a low-cost, customizable, lightweight operating system for high devices without developing one from scratch. As a result, despite being primarily designed for phones and tablets, it has seen additional applications on televisions, consoles, digital and other electronics. Android's open nature has further encouraged a large community of developers and enthusiasts to use the open-source code as a foundation for community-driven projects, which add new features for advanced users or bring Android to devices which were officially, released running other operating systems.

Android's share of the global smartphone market, led by Samsung products, was $64 \%$ in March 2013.In July 2013 there were 11,868 different models of Android device, scores of screen sizes and eight OS versions simultaneously in use. The operating system's success has made it a target for patent litigation as part of the so-called "smartphone wars" between technology companies. As of May 2013, 48 billion apps have been installed from the Google Play store, and as of September 3, 2013, there have been 1 billion Android devices activated.

\subsubsection{Applications of Android}

Android has a growing selection of third party applications, which can be acquired by users either through an app store such as Google or the Amazon, or by downloading and installing the application's APK file from a third-party site. The Play Store application allows users to browse, download and update apps published by Google and third-party developers. Applications are developed in the Java language using the Development Kit (SDK). The SDK includes a comprehensive set of development tools, including a debugger, software, a handset emulator based on QEMU, documentation, sample code, and tutorials. The officially supported integrated (IDE) is Eclipse using the Android Development Tools (ADT) plugin. Other development tools are available, including a Native for applications or extensions in $\mathrm{C}$ or $\mathrm{C}++$, Google App Inventor, a visual environment for novice programmers, and various cross.

6.2.3 Application layout

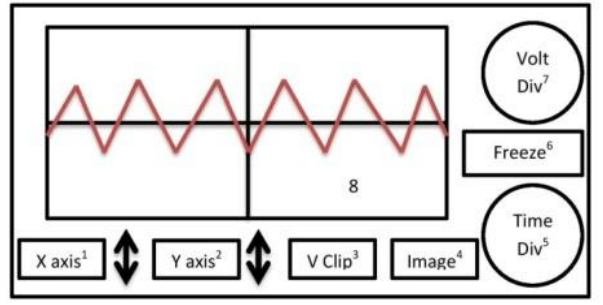

Fig 4: Application layout of Wireless Oscilloscope Android Application

6.2.3.1 X-axis Button (Horizontal position control)

It is used to move the producing graphical waveform along $\mathrm{X}$-axis (horizontally).

\subsubsection{Y-axis Button (Vertical position control)}

It is used to move the producing graphical waveform along $\mathrm{Y}$-axis (vertically).

\subsubsection{V Clip Button}

It is used to save producing waveform as a video clip.

\subsubsection{Image}

It is used to save producing waveform as an image (.jpeg)

\subsubsection{Time Div}

It is used to observe waveform as in required Time division. For example: $2 \mathrm{~ms}$, $4 \mathrm{~ms}$, Etc.

\subsubsection{Freeze}

It is used to freeze the moving pictures (i.e.) to stop waveform and to show as still image.

\subsubsection{Voltage Div}

It is used to observe waveform as in required Voltage division. For example: 2v, 4v, Etc.

\subsubsection{Display}

It is the portion where graphical waveform is produced and it is provided with multi zoom.

\section{PROTOTYPE OF PROPOSED SYSTEM}

Fig 5: Prototype of the Wireless Oscilloscope Android

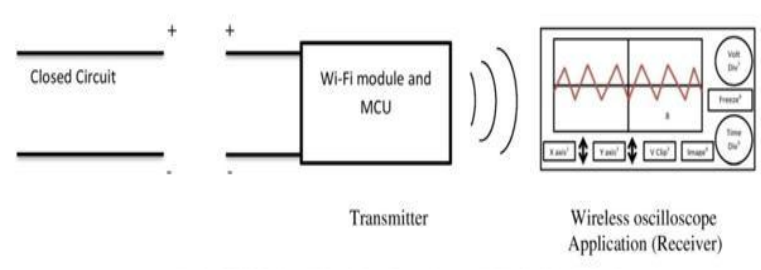

Application

\section{OPERATION}

The Wi-Fi transmitter is connected to the end of the closed circuit. Then Wi-Fi connection is turned ON in the android smartphone's or tablet computers. Wi-Fi oscilloscope application is opened and sense button is taped. Automatically output voltage is transferred as signal over radio frequency and transmitted signal is reproduced as graphical waveform (2-D moving pictures) on the screen.

We can change the voltage division and time division using corresponding varying button's for observing the waveform in corresponding voltage and time. Then we can save the obtained waveform as video clip by tapping Clip button or save it as in jpeg format by tapping screen shot button. 


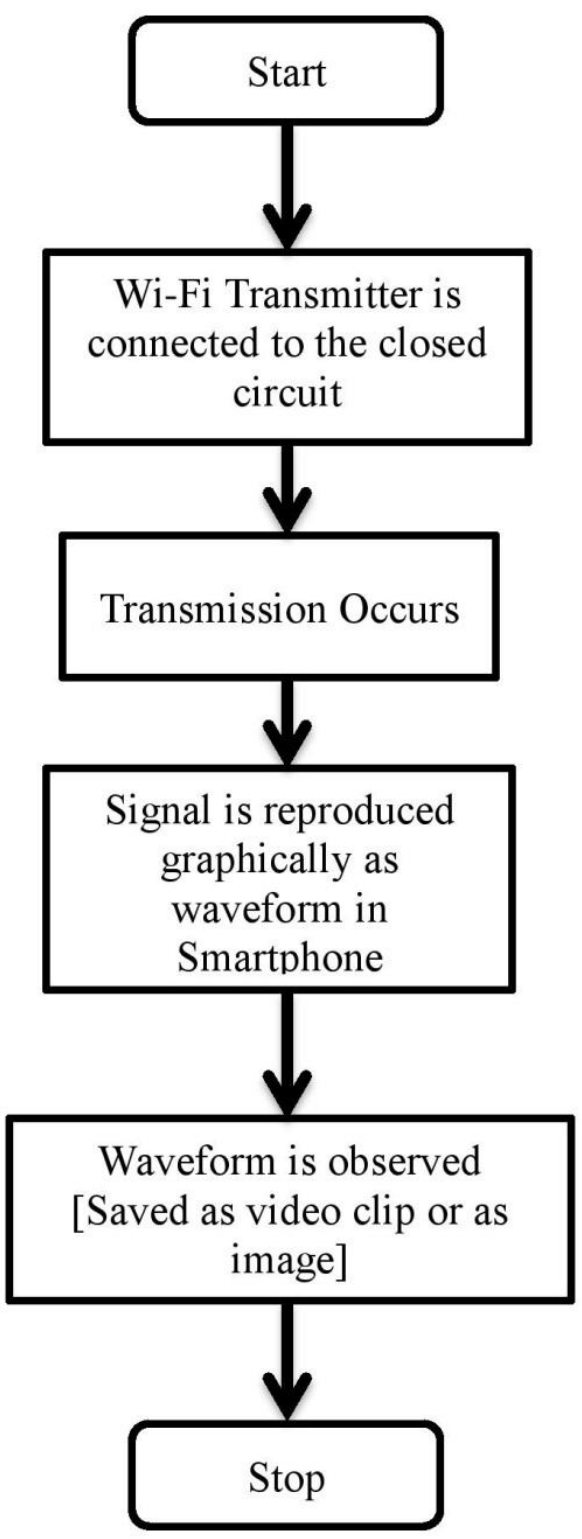

Fig 6: Flowchart of Operation occurs in the Wireless Oscilloscope Android Application

\section{CONCLUSION AND FUTURE WORK}

Wi-Fi technology is used in this application. Hence it works about $10-100 \mathrm{~m}$ at very high speed data transmission of 10-105 mbps. Wi-Fi doesn't consume high power compared to normal $\mathrm{CRO}$ and nontoxic one. Now a days, smartphone's and tablet computers are used very much among people. When this application is once installed in the smartphone or tablet computers, we can use whenever we are in need of Oscilloscope. Wi-Fi transmitter is also easily carried able in pocket. And this application also makes so easier to observe response of the circuit as graphical waveform in form of a video clip or image. This technology can also be linked with cloud computing. It will be ecologically sustainable than existing system. It will be helpful to our Indian economy indirectly.

\section{ACKNOWLEDGEMENT}

Our sincere thanks to our Professors Mr.Gnanaprakasham, Dept. of CSE, Angel College of Engineering and Technology, Tirupur-641665 and Mr. Nitin Gupta, CEO of Autorotics Technologies, Delhi who have helped us on our ideas technically throughout the preparation of this paper. Our special thanks to god, our beloved parents and friends who supported us all the time.

\section{REFERENCES}

1] "Wi-Fi (wireless networking technology)".Encyclopedia Britannica. Retrieved 2010-02-03.

[2] "Android Code Analysis". Retrieved June 6, 2012

[3] "Philosophy and Goals".Android Open Source Project.Google.Retrieved 2012-04-21.

[4] Augarten, Stan (1983)."The Most Widely Used Computer on a Chip: The TMS 1000".State of the Art: A Photographic History of the Integrated Circuit(New Haven and New York: Ticknor \& Fields).ISBN 0-89919-195-9. Retrieved 2009-12-23

[5] "The History of Java Technology". Retrieved October 6, 2012.

[6] Gosling, James; Joy, Bill; Steele, Guy; and Bracha, Gilad."The Java Language Specification, 2nd Edition".

7] Adam Osborne, An Introduction to Microcomputers Volume 1: Basic Concepts, Osborne-McGraw Hill Berkeley California USA, 1980ISBN 0-931988-34-9pp. 116-126. 